\title{
Instantaneous Bethe-Salpeter Equation and Its Exact Solution
}

\author{
Chao-Hsi Chang ${ }^{1,2}$, Jiao-Kai Chen ${ }^{2,3}$, Xue-Qian $\mathrm{Li}^{1,4}$ and Guo-Li Wang ${ }^{4,5}$ \\ 1 CCAST (World Laboratory), P.O. Box 8730, Beijing 100080, China* \\ 2 Institute of Theoretical Physics, Chinese Academy of Sciences, P.O. Box 2735, Beijing 100080, China \\ 3 Graduate School of the Chinese Academy of Sciences, Beijing 100039, China \\ 4 Department of Physics, NanKai University, TianJin 300071, China \\ 5 Department of physics, FuJian Normal University, FuZhou 350007, China.
}

\begin{abstract}
We present an approach to solve a Bethe-Salpeter (BS) equation exactly without any approximation if the kernel of the BS equation exactly is instantaneous, and take positronium as an example to illustrate the general features of the solutions. As a middle stage, a set of coupled and self-consistent integration equations for a few scalar functions can be equivalently derived from the BS equation always, which are solvable accurately. For positronium, precise corrections to those of the Schrödinger equation in order $v$ (relative velocity) in eigenfunctions, in order $v^{2}$ in eigenvalues, and the possible mixing, such as that between $S(P)$ and $D(F)$ components in $J^{P C}=1^{--}\left(J^{P C}=2^{++}\right)$states as well, are determined quantitatively. Moreover, we also point out that there is a problematic step in the classical derivation which was proposed first by E.E. Salpeter. Finally, we emphasize that for the effective theories (such as NRQED and NRQCD etc) we should pay great attention on the corrections indicated by the exact solutions.
\end{abstract}

PACS numbers: 11.10.St, 36.10.Dr, 12.20.Ds

Keywords: instantaneous BS equation, exact solutions, positronium, relativistic corrections,

Bethe-Salpeter (BS) equation [1] is a very good tool to treat various bound state systems. For a fermionantifermion binding system, the BS equation is written as follows,

$$
\left(\not p_{1}-m_{1}\right) \chi_{P}(q)\left(\not p_{2}+m_{2}\right)=i \int \frac{d^{4} k}{(2 \pi)^{4}} V(P, q, k) \chi_{P}(k)
$$

where $\chi_{P}(q)$ is the BS wave function, $P$ is the total momentum, $q$ is relative momentum, and $V(P, k, q)$ is the so-called BS kernel between the electron and positron in the bound state, $p_{1}, p_{2}$ are the momenta of the constituent electron 1 and positron 2, respectively. The total momentum $P$ and the relative momentum $q$ are related to the momenta of the two quarks as follows: $p_{1}=$ $\alpha_{1} P+q, \quad \alpha_{1}=\frac{m_{1}}{m_{1}+m_{2}}, \quad p_{2}=\alpha_{2} P-q, \quad \alpha_{2}=\frac{m_{2}}{m_{1}+m_{2}}$.

If the kernel of the four-dimensional BS equation, $V(P, k, q)$, in $\vec{P}=0$ frame (C.M.S) of the concerned bound state, has the behavior: $\left.V(P, q, k)\right|_{\vec{P}=0}=V(\vec{q}, \vec{k})$, the BS equation is called as 'instantaneous one', and may be derived to a Schrödinger equation accordingly, that was firstly realized by E.E. Salpeter [2] .

In Coulomb gauge, the transverse photon exchange is considered as higher order, so at the lowest order the BS equation for positronium has the kernel $\left.V(P, q, k)\right|_{\text {postronium }}=\gamma^{0} V_{v} \gamma^{0}=-\gamma^{0} \frac{4 \pi \alpha}{(\vec{q}-\vec{k})^{2}} \gamma^{0}$ only, that is instantaneous, so we take positronium as an example to pursue exact solutions of the BS equation. It certainly is interesting that the relativistic corrections, including the possible mixing, such as that between $S$ and $D$ components in $J^{P C}=1^{--}$state and that between $P$ and $F$

\footnotetext{
${ }^{*}$ Not post-mail address.
}

components in $J^{P C}=2^{++}$state etc, will be fully fixed by the instantaneous BS equation. Here we report that as the case of positronium, an instantaneous BS equation can be really solved without any approximation, and outline the approach briefly. We derive the instantaneous $\mathrm{BS}$ equation (the BS wave function in 4 spin structure) into a set of coupled and self-consistent integration equations for its components (scalar functions) without any approximation, and solve them numerically. The accuracy of the solutions can be contral at all. The results are discussed finally. While the details are put in Refs. [6, []].

The approach which we show here mainly is to follow E.E. Salpeter derivation but without any approximation.

As done in Ref. 2] and in book 3], the instantaneous BS wave function $\varphi_{P}(\vec{q})$ is introduce:

$$
\varphi_{P}(\vec{q}) \equiv i \int \frac{d q^{0}}{2 \pi} \chi_{P}\left(q^{0}, \vec{q}\right)
$$

then the BS equation Eq 1 is re-written

$$
\chi_{P}\left(q^{0}, \vec{q}\right)=S_{f}^{(1)}\left(p_{1}^{\mu}\right) \eta(\vec{q}) S_{f}^{(2)}\left(-p_{2}^{\mu}\right),
$$

where $S_{f}^{(1)}\left(p_{1}\right)$ and $S_{f}^{(2)}\left(-p_{2}\right)$ are the propagators of the fermion and anti-fermion respectively and the integrated 'BS-nut' $\eta(\vec{q}) \equiv \int \frac{d^{3} \vec{k}}{(2 \pi)} V(\vec{q}, \vec{k}) \varphi_{P}(\vec{k})$. For general applications, we keep $m_{1} \neq m_{2}$ at this moment, although final application in this paper is to positronium $m_{1}=m_{2}$. The propagators can be decomposed as:

$$
-i J S_{f}^{(i)}\left(J p_{i}^{\mu}\right)=\frac{\Lambda_{i}^{+}(\vec{q})}{J q^{0}+\alpha_{i} M-\omega_{i}+i \epsilon}+\frac{\Lambda_{i}^{-}(\vec{q})}{J q^{0}+\alpha_{i} M+\omega_{i}-i \epsilon},
$$

with $\omega_{i}=\sqrt{m_{i}^{2}+\vec{q}^{2}}, \quad \Lambda_{i}^{ \pm}(\vec{q})=\frac{1}{2 \omega_{i}}\left[\gamma^{0} \omega_{i} \pm J\left(m_{i}+\vec{\gamma}\right.\right.$. $\vec{q})$, where $J=1$ for the $\operatorname{quark}(i=1)$ and $J=-1$ for 
the $\operatorname{anti-quark}(i=2)$. It is easy to check

$$
\begin{gathered}
\Lambda_{i}^{ \pm}(\vec{q})+\Lambda_{i}^{\mp}(\vec{q})=\gamma^{0}, \\
\Lambda_{i}^{ \pm}(\vec{q}) \gamma^{0} \Lambda_{i}^{\mp}(\vec{q})=0, \quad \Lambda_{i}^{ \pm}(\vec{q}) \gamma^{0} \Lambda_{i}^{ \pm}(\vec{q})=\Lambda_{i}^{ \pm}(\vec{q}),
\end{gathered}
$$

Namely $\Lambda^{ \pm}$are 'energy' projection operators and complete. With them for below discussions let us define $\varphi_{P}^{ \pm \pm}(\vec{q})$ as:

$$
\varphi_{P}^{ \pm \pm}(\vec{q}) \equiv \Lambda_{1}^{ \pm}(\vec{q}) \gamma^{0} \varphi_{P}(\vec{q}) \gamma^{0} \Lambda_{2}^{ \pm}(\vec{q}) .
$$

Because of the completeness of the projectors $\Lambda^{ \pm}$, for the BS wave function $\varphi_{P}(\vec{q})$, we have: $\varphi_{P}(\vec{q})=\varphi_{P}^{++}(\vec{q})+$ $\varphi_{P}^{+-}(\vec{q})+\varphi_{P}^{-+}(\vec{q})+\varphi_{P}^{--}(\vec{q})$. As done by E.E. Salpeter, to derive the instantaneous BS equation to corresponding Schrödinger equation, by carrying out a contour integration of the time-component $q^{0}$ on both sides of Eq. (3), we obtain

$$
\varphi_{P}(\vec{q})=\frac{\Lambda_{1}^{+}(\vec{q}) \eta_{P}(\vec{q}) \Lambda_{2}^{+}(\vec{q})}{\left(M-\omega_{1}-\omega_{2}\right)}-\frac{\Lambda_{1}^{-}(\vec{q}) \eta_{P}(\vec{q}) \Lambda_{2}^{-}(\vec{q})}{\left(M+\omega_{1}+\omega_{2}\right)},
$$

and applying the complete set of the projection operators $\Lambda_{i P}^{ \pm}(\vec{q})$ to Eq. (17) further, we obtain the coupled equations:

$$
\begin{gathered}
\left(M-\omega_{1}-\omega_{2}\right) \varphi_{P}^{++}(\vec{q})=\Lambda_{1}^{+}(\vec{q}) \eta_{P}(\vec{q}) \Lambda_{2}^{+}(\vec{q}), \\
\left(M+\omega_{1}+\omega_{2}\right) \varphi_{P}^{--}(\vec{q})=-\Lambda_{1}^{-}(\vec{q}) \eta_{P}(\vec{q}) \Lambda_{2}^{-}(\vec{q}), \\
\varphi_{P}^{+-}(\vec{q})=\varphi_{P}^{-+}(\vec{q})=0 .
\end{gathered}
$$

Note that because of the completeness of the projector set, Eqs. (8) 9, 10) in whole are exactly equivalent to Eq.(17) and Eq.(3).

While E.E. Salpeter [2] and the authors of [3] would like to connect the above coupled equations to the Breit equation [4, 5], so they 'combined' Eqs. (8910) into one operator equation (in C.M.S. of the bound state):

$$
\begin{gathered}
{\left[M-H_{1}(\vec{q})-H_{2}(\vec{q})\right] \varphi(\vec{q})} \\
=\Lambda_{1}^{+}(\vec{q}) \gamma^{0} \eta(\vec{q}) \gamma^{0} \Lambda_{2}^{+}(\vec{q})-\Lambda_{1}^{-}(\vec{q}) \gamma^{0} \eta(\vec{q}) \gamma^{0} \Lambda_{2}^{-}(\vec{q}),
\end{gathered}
$$

with the definitions $H_{1}(\vec{q}) \equiv m_{1} \beta+\vec{q} \cdot \vec{\alpha}, H_{2}(\vec{q}) \equiv m_{2} \beta-$ $\vec{q} \cdot \vec{\alpha}, \beta=\gamma^{0}, \vec{\alpha}=\beta \vec{\gamma}$. Namely they considered the equation Eq.(11) with the definition $H_{1,2}$ as an operator representation of the coupled equations Eqs. (8) 10). In fact, it is no correct. Eq. (111) is not fully equivalent to the coupled-equations Eqs. (8) 9 (10).

Now let us show the un-equivalence as follows.

When applying the project operator $\Lambda_{1}^{+}(\vec{q}) \gamma^{0} \otimes$ $\gamma^{0} \Lambda_{2}^{+}(\vec{q})$ to Eq. (11) we obtain Eq.(8), when applying the project operator $\Lambda_{1}^{-}(\vec{q}) \gamma^{0} \otimes \gamma^{0} \Lambda_{2}^{-}(\vec{q})$ to Eq.(11) we obtain the Eq.(9), whereas when applying $\Lambda_{1}^{ \pm}(\vec{q}) \gamma^{0} \otimes \gamma^{0} \Lambda_{2}^{\mp}(\vec{q})$ to Eq.(11), then we obtain:

$$
\begin{aligned}
& {\left[M-\omega_{1}(\vec{q})+\omega_{2}(\vec{q})\right] \varphi^{+-}(\vec{q})=0,} \\
& {\left[M+\omega_{1}(\vec{q})-\omega_{2}(\vec{q})\right] \varphi^{-+}(\vec{q})=0,}
\end{aligned}
$$

with $\omega_{1,2}=\sqrt{m_{1,2}^{2}+\vec{q}^{2}}$. When $m_{1}=m_{2}$ i.e. $\omega_{1}=\omega_{2}$, we may obtain Eq.(10) from Eq.(12) unless $M=0$ in the extremely relativistic cases, thus in the cases $m_{1}=m_{2}$, equations Eq. (12) are equivalent to Eq.(10). Whereas when $m_{1} \neq m_{2}$ i.e. $\omega_{1} \neq \omega_{2}$, the equations Eq.(12) have the 'trivial solutions' just as Eq.(10), but also have 'non-trivial solutions'. Practically when solving equations, such as Eq.(11), one would not check the projections $\Lambda_{1}^{+}(\vec{q}) \gamma^{0} \otimes \gamma^{0} \Lambda_{2}^{+}(\vec{q}), \Lambda_{1}^{-}(\vec{q}) \gamma^{0} \otimes \gamma^{0} \Lambda_{2}^{-}(\vec{q})$ and $\Lambda_{1}^{ \pm}(\vec{q}) \gamma^{0} \otimes \gamma^{0} \Lambda_{2}^{\mp}(\vec{q})$ on the solutions precisely, and generally non-trivial solutions are chosen. Especially, when further approximations are made, certain misleading may occur i.e. the obtained solutions may not satisfy the equations Eq.(10), even in the cases $m_{1}=m_{2}$ [6]. Therefore, we conclude that Eq.(12) are not fully equivalent to Eq.(10).

We will present the different consequences due to Eq.(10) and Eq.(12) more precisely and solve them respectively in Ref. [8].

In the literature the authors of Refs. 2, 3] etc made an additional assumption on the spin structure of the BS wave function e.g. the spin structure of the wave functions for $S$-wave positronium has the formulation (in C.M.S. $\vec{P}=0)$ :

$$
\varphi_{0^{-+}\left({ }^{1} S_{0}\right)}(\vec{q})=\left[\gamma_{0}+1\right] \gamma_{5} f(\vec{q})
$$

for $J^{P C}=0^{-+}\left({ }^{1} S_{0}\right)$; and

$$
\varphi_{1^{--}\left({ }^{3} S_{1}\right), \lambda}(\vec{q})=\left[\gamma_{0}+1\right] A_{\lambda} f(\vec{q})
$$

for $J^{P C}=1^{--}\left({ }^{3} S_{1}\right)$. Here $A_{\lambda} \equiv \epsilon_{\lambda}^{+} \gamma^{-}+\epsilon_{\lambda}^{-} \gamma^{+}-\epsilon_{\lambda}^{\Delta} \gamma^{\Delta}$. With the spin structure finally the bound state problem turned to solve a Schrödinger equation accordingly by taking the so-called positive-energy equation Eq.(8) only (the rests are ignored).

To solve the instantaneous BS equation Eq.(3) for positronium without any approximation, even in the case $m_{1}=m_{2}$ we 'return' to solve the Eqs. (8) 90 10). In fact, the approach here is also applicable in the cases $m_{1} \neq m_{2}$.

In $\vec{P}=0$ frame (C.M.S.), the most general formulation of the BS wave function for the bound state $J^{P C}=0^{-+}$ $\left({ }^{1} S_{0}\right)$ may be written as the follows:

$$
\begin{gathered}
\varphi^{1} S_{0}(\vec{q})=\gamma^{0} \gamma^{5} \varphi_{1}(|\vec{q}|)+\gamma^{5} \varphi_{2}(|\vec{q}|) \\
+\sqrt{\frac{4 \pi}{3}} \gamma^{5} \frac{|\vec{q}|}{2 m} E \varphi_{3}(|\vec{q}|) \gamma^{0}+\sqrt{\frac{4 \pi}{3}} \gamma^{5} \frac{|\vec{q}|}{2 m} E \varphi_{4}(|\vec{q}|)
\end{gathered}
$$

where $E \equiv\left[Y_{1-1} \gamma^{+}+Y_{11} \gamma^{-}-Y_{10} \gamma^{3}\right], \gamma^{+} \equiv-\frac{\gamma^{1}+i \gamma^{2}}{\sqrt{2}}$, $\gamma^{-}=\frac{\gamma^{1}-i \gamma^{2}}{\sqrt{2}}$ and $Y_{l m} \equiv Y_{l m}\left(\theta_{q}, \phi_{q}\right)$ are spherical harmonics. To apply the equation Eq.(10) to Eq.(15), we obtain the 'constraints' for the components of the wave function precisely:

$$
\varphi_{1}(|\vec{q}|)=-\frac{1}{2} \varphi_{4}(|\vec{q}|), \quad \varphi_{3}(|\vec{q}|)=0 .
$$


TABLE I: The expansion coefficients $C_{i, n l}^{(j)}$ for the $J^{P C}=0^{-+}$ states.

\begin{tabular}{|c|c|c|c|c|c|}
\hline \hline $\mathrm{NL}$ & $\mathrm{WF}$ & $C_{i, 1 S}^{(j)}$ & $C_{i, 2 S}^{(j)}$ & $C_{i, 3 S}^{(j)}$ & $C_{i, 4 S}^{(j)}$ \\
\hline$E^{(1)}$ & $f_{1}^{(1)}$ & -0.707104 & $8.934 \mathrm{D}-6$ & $4.225 \mathrm{D}-6$ & $2.629 \mathrm{D}-6$ \\
\cline { 2 - 6 }$\left(1^{1} S_{0}\right)$ & $f_{2}^{(1)}$ & -0.707109 & $6.962 \mathrm{D}-6$ & $3.206 \mathrm{D}-6$ & $1.979 \mathrm{D}-6$ \\
\hline$E^{(2)}$ & $f_{1}^{(2)}$ & $5.031 \mathrm{D}-6$ & 0.707106 & $-7.246 \mathrm{D}-6$ & $-3.571 \mathrm{D}-6$ \\
\cline { 2 - 6 }$\left(2^{1} S_{0}\right)$ & $f_{2}^{(2)}$ & $7.004 \mathrm{D}-6$ & 0.707107 & $-6.567 \mathrm{D}-6$ & $-3.169 \mathrm{D}-6$ \\
\hline$E^{(3)}$ & $f_{1}^{(3)}$ & $2.188 \mathrm{D}-6$ & $5.898 \mathrm{D}-6$ & 0.707107 & $-6.768 \mathrm{D}-6$ \\
\cline { 2 - 6 }$\left(3^{1} S_{0}\right)$ & $f_{2}^{(3)}$ & $3.207 \mathrm{D}-6$ & $6.577 \mathrm{D}-6$ & 0.707107 & $-6.425 \mathrm{D}-6$ \\
\hline \hline
\end{tabular}

$\varphi_{3}, \varphi_{4}$ in Eq.(15) are replaced with Eq. (16), then the relativistic wave function of the state $0^{-+}$contain two independent components $\varphi_{1}, \varphi_{2}$ only. With straightforward calculations, from Eqs. (8) for positronium, finally we obtain the two coupled radius equations:

$$
\begin{gathered}
(M-2 m) f_{1}+2 m f_{1}-2 m f_{2}= \\
-\frac{\alpha_{s}}{\pi} \frac{m}{\omega} \int \frac{|\vec{k}|}{|\vec{q}|} d|\vec{k}| Q_{0} f_{2}, \\
(M-2 m) f_{2}-2 \frac{\omega^{2}}{m} f_{1}+2 m f_{2}= \\
-\frac{\alpha_{s}}{\pi} \frac{m}{\omega} \int \frac{|\vec{k}|}{|\vec{q}|} d|\vec{k}|\left(Q_{0}+\frac{|\vec{q}||\vec{k}|}{m^{2}} Q_{1}\right) f_{1},
\end{gathered}
$$

where $Q_{n} \equiv Q_{n}\left(\frac{|\vec{q}|^{2}+|\vec{k}|^{2}}{2|\vec{q}||\vec{k}|}\right) \quad(n=0,1, \cdots)$ are the $n$ the Legendre functions of the second kind. Here to shorten the notations we have introduced $f_{1,2}: \varphi_{1,2}(|\vec{q}|) \equiv f_{1,2}$ and $\omega=\omega_{1,2}$, and the angular integrations have been carried out precisely. Now without any approximation a self-consistent problem, two coupled equations Eq. (17) for two independent scalar functions $f_{1,2}$, is reached to.

Then we further solve the coupled equations Eq. (17) numerically by expanding the components $f_{1,2}$ and the equations in terms of the bases of the exact solutions of the Schrödinger equation [9], and then diagonalizing the matrix equations.

The final results for the eigenvalues $E^{(j)}, j=1,2, \cdots$ :

$$
\begin{gathered}
E^{(1)}=-6.8026952534 ; \quad E^{(2)}=-1.70069524809 ; \\
E^{(3)}=-0.75586715480 ; \quad E^{(4)}=-0.42517586843 ;(.18)
\end{gathered}
$$

and the corresponding eigenfunctions

$$
f_{i}^{(j)}(|\vec{q}|)=\sum_{n l} C_{i, n l}^{(j)} \cdot R_{n l}(|\vec{q}|),
$$

where $(j)$ denotes those correspond to the $j$ th eigenvalue Eq.(18), $R_{n l}(|\vec{q}|)(n l=1 S, 2 S, 2 P, 3 S, 3 P, \cdots)$ are the Schrödinger radius solutions in momentum representation for the positronium and their precise expression can be found in Ref.9]. For the low-laying solutions the coefficients $C_{i, n l}^{(j)}(n l \leq 4 S)$ are put in TABLE I and $C_{i, n l}^{(j)} \leq 10^{-6}(n l>4 S)$. Based on the values in TABLE I, up to the order $v$ accuracy the eigenfunctions for $J^{P C}=0^{-+}\left({ }^{1} S_{0}\right)$ states, in fact, can be simplified to write as

$$
\varphi^{1 S_{0}}(\vec{q}) \simeq\left\{\gamma^{0}+1+\sqrt{\frac{4 \pi}{3}} E \gamma^{0} \frac{|\vec{q}|}{m}\right\} f \gamma^{5}
$$

with $f_{1}(\vec{q}) \simeq f_{2}(\vec{q}) \equiv f=R_{n(l=0)}(|\vec{q}|)$.

Let us now solve the equations for the $1^{--}$states.

Similar to $0^{-+}$states, we start with the most general BS wave function, and have the constraints Eq. (10) applied, then the BS wave function becomes the form:

$$
\begin{gathered}
\varphi_{1^{--}, \lambda}(\vec{q})=\left(2 \sqrt{\frac{\pi}{3}} C_{\lambda}+A_{\lambda}\right) f_{1} \\
+\left(-2 \sqrt{\frac{\pi}{3}} D_{\lambda} \gamma^{5}+A_{\lambda} \gamma^{0}\right) f_{2}+\left(-2 \sqrt{\frac{5}{3}} C_{\lambda}+B_{\lambda}\right) f_{3} \\
+\left(\sqrt{\frac{5}{3}} D_{\lambda} \gamma^{5}-B_{\lambda} \gamma^{0}\right) f_{4}
\end{gathered}
$$

where $A_{\lambda}$ is the same as in Eq. 114 and

$$
\begin{gathered}
B_{\lambda} \equiv \sqrt{6} \epsilon_{\lambda}^{+} \gamma^{+} Y_{2-2}-\sqrt{3}\left(\epsilon_{\lambda}^{+} \gamma^{\Delta}+\epsilon_{\lambda}^{\Delta} \gamma^{+}\right) Y_{2-1} \\
+\left(\epsilon_{\lambda}^{+} \gamma^{-}+\epsilon_{\lambda}^{-} \gamma^{+}+2 \epsilon_{\lambda}^{\Delta} \gamma^{\Delta}\right) Y_{20} \\
-\sqrt{3}\left(\epsilon_{\lambda}^{-} \gamma^{\Delta}+\epsilon_{\lambda}^{\Delta} \gamma^{-}\right) Y_{21}+\sqrt{6} \epsilon_{\lambda}^{-} \gamma^{-} Y_{22}, \\
C_{\lambda} \equiv \frac{|\vec{q}|}{m}\left[\epsilon_{\lambda}^{+} Y_{1-1}+\epsilon_{\lambda}^{-} Y_{11}-\epsilon_{\lambda}^{\Delta} Y_{10}\right], \\
D_{\lambda} \equiv \frac{|\vec{q}|}{m}\left[\left(\epsilon_{\lambda}^{-} \gamma^{\Delta}-\epsilon_{\lambda}^{\Delta} \gamma^{-}\right) Y_{11}+\left(\epsilon_{\lambda}^{\Delta} \gamma^{+}-\epsilon_{\lambda}^{+} \gamma^{\Delta}\right) Y_{1-1}\right. \\
\left.+\left(\epsilon_{\lambda}^{+} \gamma^{-}-\epsilon_{\lambda}^{-} \gamma^{+}\right) Y_{10}\right] .
\end{gathered}
$$

Here $\Delta \equiv 0$ is introduced for the $0^{\text {th }}$ component to avoid confusions. From Eq. 21), we may see clearly that $f_{3,4}$ correspond to $D$-wave components while $f_{1,2}$ to $S$-wave ones. The wave function now has four independent components $f_{i}(i=1, \ldots, 4)$.

From Eqs. (89) and to carry out the angular integration in the equations, finally we obtain the coupled radius equations:

$$
\begin{gathered}
(M-2 m) f_{1}=\frac{2 \sqrt{5}}{3 \sqrt{\pi}} \frac{|\vec{q}|^{2}}{m} f_{4}-2 m f_{1}-\frac{2 m^{2}+4 \omega^{2}}{3 m} f_{2} \\
-\frac{\alpha}{\pi} \int \frac{|\vec{k}|}{|\vec{q}|} d|\vec{k}|\left[\sqrt{\frac{5}{9 \pi}} \frac{|\vec{q}||\vec{k}|}{m \omega} Q_{1} f_{4}-\left(\frac{2}{3} \frac{|\vec{q}||\vec{k}|}{m \omega} Q_{1}+\frac{m}{\omega} Q_{0}\right) f_{2}\right], \\
(M-2 m) f_{2}=\frac{2 \sqrt{5}}{3 \sqrt{\pi}} \frac{|\vec{q}|^{2}}{m} f_{3}-\frac{2}{3} \frac{2 m^{2}+\omega^{2}}{m} f_{1}-2 m f_{2} \\
-\frac{\alpha}{\pi} \int \frac{|\vec{k}|}{|\vec{q}|} d|\vec{k}|\left[\frac{1}{3} \sqrt{\frac{5}{\pi}} \frac{|\vec{q}||\vec{k}|}{m \omega} Q_{1} f_{3}-\left(\frac{1}{3} \frac{|\vec{q}||\vec{k}|}{m \omega} Q_{1}+\frac{m}{\omega} Q_{0}\right) f_{1}\right], \\
(M-2 m) f_{3}=-2 m f_{3}+\frac{2}{3} \frac{2 m^{2}+\omega^{2}}{m} f_{4}-\frac{4 \sqrt{\pi}}{3 \sqrt{5}} \frac{|\vec{q}|^{2}}{m} f_{2} \\
-\frac{\alpha}{\pi} \int \frac{|\vec{k}|}{|\vec{q}|} d|\vec{k}|\left[\left(\frac{|\vec{q}||\vec{k}|}{3 m \omega} Q_{1}+\frac{m}{\omega} Q_{2}\right) f_{4}-\frac{2 \sqrt{\pi}}{3 \sqrt{5}} \frac{|\vec{q}||\vec{k}|}{m \omega} Q_{1} f_{2}\right],
\end{gathered}
$$




$$
\begin{gathered}
(M-2 m) f_{4}=\frac{1}{3} \frac{2 m^{2}+4 \omega^{2}}{m} f_{3}-2 m f_{4}-\frac{4 \sqrt{\pi}}{3 \sqrt{5}} \frac{|\vec{q}|^{2}}{m} f_{1} \\
-\frac{\alpha}{\pi} \int \frac{|\vec{k}|}{|\vec{q}|} d|\vec{k}|\left[\left(\frac{m}{\omega} Q_{2}+\frac{2}{3} \frac{|\vec{q}||\vec{k}|}{m \omega} Q_{1}\right) f_{3}\right. \\
\left.-\frac{2 \sqrt{\pi}}{3 \sqrt{5}} \frac{|\vec{q}||\vec{k}|}{m \omega} Q_{1} f_{1}\right] .
\end{gathered}
$$

Four coupled equations for four independent components $f_{1,2,3,4}$ is the request of the $S$ - $D$ wave mixing and a selfconsistent problem is reached.

We solve the Eqs. (22) numerically with the same method as adopted in the case for $0^{-+}$, and obtain the results:

$$
\begin{array}{ll}
E^{(1)}\left(1^{3} S_{1}\right)=-6.8027275 ; & E^{(2)}\left(2^{3} S_{1}\right)=-1.7007008 \\
E^{(3)}\left(3^{3} S_{1}\right)=-0.7558690 ; & E^{(4)}\left(3^{3} D_{1}\right)=-0.7558729
\end{array}
$$$$
\cdots
$$

for eigenvalues, while the table of the coefficients $C_{i, n l}^{(j)}$ for the eigenfunctions is too big to present here. Instead, we present them here only up to the order $v$ accuracy as in the case for $0^{-+}$.

For $J^{P C}=1^{--}\left({ }^{3} S_{1}\right)$ states we can simplify: $f_{1}(\vec{q}) \simeq$ $-f_{2}(\vec{q}) \equiv f=R_{n(l=0)}(|\vec{q}|)$ and $f_{3}(\vec{q}) \simeq f_{4}(\vec{q}) \simeq 0$, i.e.

$$
\begin{aligned}
& { }^{\varphi^{3} S_{1}, \lambda}(\vec{q}) \simeq\left[\left(2 \sqrt{\frac{\pi}{3}} \frac{|\vec{q}|}{m} C_{\lambda}+A_{\lambda}\right)\right. \\
& \left.-\left(-2 \sqrt{\frac{\pi}{3}} \frac{|\vec{q}|}{m} D_{\lambda} \gamma^{5}+A_{\lambda} \gamma^{0}\right)\right] f .
\end{aligned}
$$

For $J^{P C}=1^{--}\left({ }^{3} D_{1}\right)$ states we can simplify: $f_{1}(\vec{q}) \simeq$ $f_{2}(\vec{q}) \simeq 0$ and $f_{3}(\vec{q}) \simeq f_{4}(\vec{q}) \equiv f=R_{n(l=2)}(|\vec{q}|)$, i.e.

$$
\begin{gathered}
\varphi^{\varphi^{3} D_{1}, \lambda}(\vec{q}) \simeq\left[\left(-2 \sqrt{\frac{5}{3}} \frac{|\vec{q}|}{m} C_{\lambda}+B_{\lambda}\right)\right. \\
\left.+\left(\sqrt{\frac{5}{3}} \frac{|\vec{q}|}{m} D_{\lambda} \gamma^{5}-B_{\lambda} \gamma^{0}\right)\right] f
\end{gathered}
$$

The accurate coefficients $C_{i, n, ~}^{(j)}$ for $J^{P C}=1^{--}$can be found in our achieved paper [7].

We may see that the exact solutions obtained here for the wave function contain the order $v=\frac{|\vec{q}|}{m}$ corrections to the approximate ones obtained by E.E. Salpeter approaches (described as above), namely, the terms in Eqs. 20] 24 25) explicitly proportional to $v$. The corrections will cause the normalization for the wave function a change in order $v^{2}$ (the detail in 7 ). The most interesting fact is the exact solutions for $1^{--}$states present the possible $S-D$ wave mixing. To compare the eigenvalues Eq.(18) for $0^{-+}$and Eq.(23) for $1^{--}$ with those of the Schrödinger solution (the degenerate ones without any splitting), we may see the 'hyperfine' splitting for spin singlet and triplet states which is in order of $\alpha^{2}(v \simeq \alpha)$, and there is a remarkable splitting between $n\left[{ }^{3} S_{1}\right]$ and $n\left[{ }^{3} D_{1}\right](n \geq 3)$ for $1^{--}$states $E^{(3)}\left(3^{3} S_{1}\right) \geq E_{3}^{S c h} \geq E^{(4)}\left(3^{3} D_{1}\right)$ and $\Delta E \simeq \alpha^{2}$ due to the fact that the BS equation Eq.(3) is relativistic.

Note that the approach we present here may be applicable for all the instantaneous BS equations and can obtain the exact solutions, while the features of the exact solutions for positronium described above may be different for an instantaneous BS equation with a different kernel from that of Coulomb interaction.

Based on a specific problem of positronium, we have learnt that the wave functions contain quite great relativistic corrections thus for effective theories, such as NRQED [10] (and NRQCD [11]), the relativistic corrections from the wave functions should be considered carefully if the relevant calculations declare the relativistic effects are taken into account. For NRQED (NRQCD) etc, the wave function effects may be involved either at matching the underlined theory to the effective one or into the non-pertubative matrix elements. Moreover the problems, such as gauge invariance for the matrix elements and $E 1$ (electrical dipole) radiative transitions etc, are sensitive to the effects, the exact solutions are crucial [6].

Acknowledgement: The author (C.H. Chang) would like to thank Stephen L. Adler for valuable discussions and to thank Xiangdong Ji for very useful discussions. The authors would like to thank Tso-Hsiu Ho and Cheng-Rui Ching for valuable suggestion and encouragement. This work was supported in part by the National Natural Science Foundation of China.
[1] E.E. Salpeter and H.A. Bethe, Phys. Rev. 84, 1232 (1951).

[2] E.E. Salpeter, Phys. Rev. 87, 328 (1952).

[3] C. Itzykson and J. Zuber, Quantum Field Theory, McGRAW-HILL Int. Book Company.

[4] G. Breit, Phys. Rev. 34, 553 (1929).

[5] G. Breit and G.E. Brown, Phys. Rev. 74, 1278 (1948); G.E. Brown and D.G. Ravenhall, Proc. Roy. Soc. (London), A208, 552 (1951).

[6] Chao-Hsi Chang, Jiao-Kai Chen and Guo-Li Wang, hep-th/0312250

[7] Chao-Hsi Chang, Jiao-Kai Chen, Xue-Qian Li and Guo-
Li Wang, in preparation.

[8] Chao-Hsi Chang, Jiao-Kai Chen, Xue-Qian Li and GuoLi Wang, in preparation.

[9] H.A. Bethe and E.E. Salpeter, Quantum Mechanics of One- and Two-Electron Atoms, Springer-Verlag, 1957.

[10] W.E. Caswell and G.P. Lepage, Phys. lett. 167, 437 (1986).

[11] Geoffrey T. Bodwin, Eric Braaten and G. Peter Lepage, Phys. Rev. D 51, 1125 (1995); Erratum ibid 55, 5835 (1997). 